\title{
Multidrug-resistant malaria and the impact of mass drug administration
}

This article was published in the following Dove Press journal: Infection and Drug Resistance

\section{Janie Anne Zuber \\ Shannon Takala-Harrison}

Division of Malaria Research, Institute for Global Health, University of Maryland School of Medicine, Baltimore, MD, USA
Correspondence: Shannon

Takala-Harrison

Division of Malaria Research, Institute

for Global Health, University of Maryland

School of Medicine, 685 W Baltimore

Street, HSF2-480, Baltimore, MD 2I20I,

USA

Email stakala@som.umaryland.edu

\begin{abstract}
Based on the emergence and spread throughout the Greater Mekong Subregion (GMS) of multiple artemisinin-resistant lineages, the prevalence of multidrug resistance leading to high rates of artemisinin-based combination treatment failure in parts of the GMS, and the declining malaria burden in the region, the World Health Organization has recommended complete elimination of falciparum malaria from the GMS. Mass drug administration (MDA) is being piloted as one elimination intervention to be employed as part of this effort. However, concerns remain as to whether MDA might exacerbate the already prevalent problem of multidrug resistance in the region. In this review, we briefly discuss challenges of MDA, the use of MDA in the context of multidrug-resistant malaria, and the potential of different drug combinations and drug-based elimination strategies for mitigating the emergence and spread of resistance.

Keywords: malaria, drug resistance, mass drug administration
\end{abstract}

\section{Introduction}

The malaria burden has declined dramatically over the last 10-15 years in many malaria-endemic areas, owing in part to a massive global scale-up of highly efficacious artemisinin-based combination therapies (ACTs). ${ }^{1}$ This progress has led to a renewed call for global malaria eradication, accompanied by a large global investment in malaria control and elimination. ${ }^{2}$ Artemisinin-resistant Plasmodium falciparum was first reported in western Cambodia ${ }^{3,4}$ and subsequently in other Greater Mekong Subregion (GMS) countries. ${ }^{5-7}$ An aggressive regional campaign to eliminate artemisinin-resistant $P$. falciparum malaria while it is still confined to limited areas of the GMS was recommended in 2015 by the World Health Organization (WHO). ${ }^{8}$ This recommendation was based, in part, on the evidence that multiple artemisininresistant parasite lineages had both emerged independently in different countries as well as spread between countries in the GMS. ${ }^{9,10}$

The principle tools for malaria elimination in the GMS are prompt diagnosis and treatment of clinical cases using rapid diagnostic tests (RDT) and artemisinin-based combination therapies (ACTs), insecticide-treated nets, and enhanced surveillance. ${ }^{8}$ Drug-based interventions such as mass drug administration (MDA) are also being considered. ${ }^{11}$ MDA is a drug treatment of all members of a population at risk, without individual testing, in an attempt to eliminate all malaria infections, including asymptomatic infections that would not otherwise be treated. MDA is a controversial approach that has been used for malaria control and elimination with variable success. ${ }^{11}$ One of the major concerns is the potential impact of MDA on the emergence and spread 
of antimalarial drug resistance. In this review, we discuss the challenges of MDA for malaria, the use of MDA in the context of multidrug-resistant malaria, and the potential of different drug combinations and drug-based elimination strategies for mitigating the emergence and spread of resistance.

\section{Challenges of MDA}

The rationale for MDA is described in one of the first documented trials of "mass treatment" with a drug, plasmoquine, ${ }^{12}$ in labor camps on a rubber plantation in West Africa. MDA was implemented with the intent to interrupt transmission of malaria parasites by reducing the number of individuals carrying gametocytes:

[...] most of the mischief is done by a few effective carriers. The logical thing would be to find the carriers and treat them. However, such persons often give little or no indication of illness [...] any one may become a good carrier of gametocytes. ${ }^{12}$

Despite a precipitous fall in infection rates after the mass treatment in this study, infections continued, and the investigators were unable to completely interrupt transmission. It was suspected that some carriers of gametocytes may have escaped treatment and/or some infected mosquitoes might have come in from untreated parts of the plantation, ultimately raising doubts of the efficacy as well as of the cost-effectiveness of MDA in this setting.

MDA for malaria has sparked controversy from its very beginning. From the massive distribution of free quinine in Italy in the early $1900 s^{13}$ to the twice weekly proguanil proffered as prophylaxis in Kenya during the mid-1900s, ${ }^{14}$ to the Garki Project in Nigeria, ${ }^{15}$ and to the multidrug combinations utilized in The Gambia toward the end of the 20th century, ${ }^{16}$ malaria MDA has had initially promising results, with significant reductions in parasite prevalence, but this impact has often proved only transient.

Generally, malaria transmission has not been interrupted, unless MDA was complemented with vector control, rigorous surveillance, and timely outbreak responses, especially in settings characterized by high transmission over wide areas. ${ }^{11}$ Malaria transmission was permanently interrupted on the Pacific island of Aneityum ${ }^{17}$ with simultaneous implementation of three malaria control activities: prevention using insecticide-treated bed nets, vector control using larvivorous fish, and intensive MDA using a three-drug combination. Targeted MDA - meaning MDA applied to a small risk area or population - is more likely to work where malaria risk is well circumscribed, such as on true sea islands or "islands" of malaria risk surrounded by areas with no malaria. ${ }^{18}$ The likelihood of success is very low in areas of contiguous malaria risk, with inevitable backflow of infections, unless MDA and other interventions are applied widely and simultaneously across the entire area of contiguous risk. In contrast to the success on Aneityum, malaria elimination efforts on the island of Zanzibar in the early 1970s using less intensive MDA followed by insecticide spraying at the end of the MDA campaign showed no measurable impact. ${ }^{19}$ Concomitant application of multiple approaches, in addition to the geographical isolation of targeted regions of elimination, may be essential factors contributing to the success of MDA-driven malaria elimination.

A total of 22 MDA projects conducted between 1932 and 1999 and their outcomes were summarized by von Seidlein and Greenwood. ${ }^{20}$ A 2013 Cochrane review included five more recent MDA experiences among a total of 32 MDA studies. ${ }^{21}$ Both of these evaluations concluded that although MDA initially seemed to reduce malaria parasite prevalence substantially, few studies demonstrated sustained impact of the intervention beyond the 6-month period following the final treatment dose of the MDA campaign. A subsequent review of 270 reports of MDA was able to identify only 48 MDA studies with follow-up periods sustained longer than 6 months. ${ }^{22}$ The early MDA studies exemplify challenges inherent to this strategy, including sustainability, logistics, and cost. More recent and contemporary projects demonstrate the challenges of ensuring sufficient population coverage and of obtaining data from longer follow-up periods. Overcoming these operational challenges is essential to understanding the impact of MDA campaigns.

\section{Examples of emergence of drug resistance in association with MDA Medicated salts}

During the 1950s, under the WHO global malaria eradication effort, antimalarial drugs such as chloroquine or pyrimethamine were added to table salt (commonly referred to as the Pinotti method) and had some success in initially reducing malaria prevalence in large-scale pilot programs in Southeast Asia, Africa, and South America. ${ }^{23}$ However, widespread use of the medicated salt both for cooking and for flavoring food resulted in slowly rising and highly variable antimalarial drug concentrations in the population - a milieu in which many parasites would have been exposed to subtherapeutic concentrations of antimalarial drugs. Another disadvantage of this strategy was a relative lack of coverage for infants and small children. ${ }^{24}$ Pyrimethamine-medicated salt was deployed 
in various countries, including the Netherlands, New Guinea, Brazil, and Cambodia. ${ }^{25}$ The effect of this administration was initially to decrease parasite prevalence in these areas, with a subsequent return to preimplementation parasite levels within $\sim 6$ months. Chloroquine-medicated salt was introduced into places such as Guyana ${ }^{26}$ and Uganda, ${ }^{27}$ where it seemed to be somewhat more effective than the pyrimethamine salt trials. Furthermore, resistance to pyrimethamine seemed to be induced rapidly, whereas resistance to chloroquine seemed to arise less readily, a phenomenon that was later shown in genetic studies to be related to the complexity of the resistance mechanism. ${ }^{28}$ However, it has since been noted repeatedly that resistance to chloroquine first arose in those same places where it had been added to the salt. ${ }^{29}$

\section{Pyrimethamine resistance in Tanzania}

Field studies mapping the rapid emergence of pyrimethamine resistance were conducted by Clyde and Shute in Tanzania in the $1950 \mathrm{~s} .{ }^{30}$ Monthly administration of subcurative doses of pyrimethamine precipitated treatment failures that were noted as early as the third monthly dose and continued to increase, all the more quickly when dosing frequency was increased to weekly. Resistance was shown to emerge rapidly and focally, with its prevalence correlated with its radial distance from the original focus of resistance. Locally applied drug pressure resulted in a directly contiguous spread of drug-resistant parasites. A similar effect was also reported from mass pyrimethamine administration schemes in Ghana and Nigeria. ${ }^{31}$

\section{MDA in the context of multidrug- resistant malaria}

Nearly all malaria-endemic countries have replaced resistance-compromised former first-line antimalarial drugs with ACTs, resulting in reduced malaria burden in many areas. ${ }^{1}$ Reports of emerging artemisinin resistance began to surface along the Thailand-Cambodia border $\sim 10$ years ago, ${ }^{3,4}$ threatening to derail recent progress in eliminating and eventually eradicating malaria. With increasing rates of treatment failure of ACTs such as artesunate-mefloquine and artemether-lumefantrine, ${ }^{32,33}$ dihydroartemisinin-piperaquine became the drug of choice to treat artemisinin-resistant malaria in Cambodia. Dihydroartemisinin-piperaquine is also being used in large-field evaluations of targeted MDA to eliminate malaria within the GMS in hopes of arresting the spread of resistance. ${ }^{34}$

So long as the partner drug component remains effective, ACTs have retained their efficacy at treating artemisininresistant malaria. However, artemisinin-resistant parasites are essentially treated by the partner drug alone. ACT partner drugs have half-lives that are much longer than those of the rapidly cleared artemisinins, providing a window of selection during which new infections are exposed to residual, subtherapeutic levels of drug that can lead to selection of resistant parasites. ${ }^{35,36}$ Mathematical models have suggested that these windows of selection are wider than estimated based on clinical data $^{37}$ and are also wider for resistant parasites acquired from the population compared to resistant parasites that emerge de novo within a given host. ${ }^{38}$ Such a wide window of selection is of particular concern when high-level resistance already exists in the population or when high-level resistance occurs by a simple mechanism (eg, single-point mutations). For example, high-level resistance to atovaquone can result from single-point mutations in the active site of $P$. falciparum cytochrome $b .{ }^{39}$ Using mathematical modeling approaches, Maude et $\mathrm{al}^{40}$ concluded that use of atovaquone-proguanil for MDA would lead to rapid selection for high-level resistance, even after a single round of MDA, rendering subsequent rounds of treatment less effective and resulting in complete loss of efficacy within 4-5 years. These same models suggested that use of an ACT, such as dihydroartemisinin-piperaquine, would be more effective and less prone to the emergence and spread of drug resistance, owing to the milder resistance phenotype resulting from artemisininresistant mutations. However, the model assumed only a $10 \%$ prevalence of parasites with artemisinin resistance and no piperaquine resistance. In several regions of Cambodia, artemisinin-resistant mutations are approaching fixation, ${ }^{41}$ and piperaquine resistance has emerged and rapidly increased in prevalence, resulting in dihydroartemisinin-piperaquine treatment failures as high as $46 \%$ in western and northern Cambodia. ${ }^{42,43}$ The effect of MDA on the emergence and spread of drug resistance when implemented in regions with resistance to both the artemisinins and key partner drugs remains a key knowledge gap.

\section{Factors related to the emergence and spread of drug resistance Drug dose}

Selection for both de novo and pre-existing resistance is more likely to occur in the presence of subtherapeutic doses of drug. ${ }^{44,45}$ The addition of antimalarial drugs such as chloroquine and pyrimethamine to table salt led to highly variable and subtherapeutic drug levels in a large proportion of treated population, as reviewed by von Seidlein and Greenwood. ${ }^{20}$ Such indirect MDA may have contributed to the rapid acquisition and spread of resistance to these drugs. ${ }^{46}$ 
In contrast, limited data suggest that the emergence of drug resistance has not been associated with the administration of therapeutic doses of antimalarial drugs given through direct MDA programs. ${ }^{20}$ In the case of artemisinin resistance, parasites with kelch13 mutations displaying delayed parasite clearance could often still be cleared with a full course of artesunate or an ACT, ${ }^{7,47}$ suggesting that adequate dose and duration of treatment may allow clearance of all but highly resistant parasites.

\section{Level of parasitemia}

The probability of a drug-resistance mutation occurring is greater in infections with high parasite density (parasitemia). ${ }^{45,48}$ In addition, treatment failure is thought to be critical to the spread of drug-resistant parasites within a population, ${ }^{45}$ and hyperparasitemic individuals are more likely to fail treatment. ${ }^{49,50}$ Asymptomatic infections have lower levels of parasitemia and presumably are controlled by some degree of host immunity, which may aid in clearing resistant parasites ${ }^{51}$ and/or deterring resistant parasites from reaching densities that allow efficient transmission. ${ }^{52}$ As most infected individuals treated as part of MDA programs are asymptomatic and have lower parasitemia, the probability of the emergence and selection for de novo resistance may be reduced; 52 however, it is still not clear whether MDA conducted in areas with well-established resistance to both the artemisinin component and partner drug would result in the spread of resistance. The outcome would likely depend on the prevalence of parasites with high-level resistance, the ACT being used, and the drug-based elimination strategy being deployed.

\section{Resistance mechanism}

The degree of resistance resulting from a mutation event is a key determinant of whether drug-resistant parasites will be selected in favor of drug-sensitive parasites. ${ }^{45}$ If a resistance mutation results in high-level resistance, such as cytochrome $b$ mutations with atovaquone, then the probability of selecting for de novo or existing resistance is higher. However, if resistance occurs in a stepwise manner (eg, the step-wise point mutations responsible for progressively increased resistance to the antifolates) or by a complex mechanism (eg, the multiple mutations that affect the level of resistance conferred by the K76T mutation within the $P$. falciparum chloroquine-resistant transporter), ${ }^{28}$ then de novo mutations may result in low-level resistance that does not provide a large selective advantage for the parasite and that may allow elimination of mutated parasites at standard drug doses.
The latter may be the case for resistance to the artemisinins, where the genetic background on which kelch13 mutations arise influences the degree of resistance, ${ }^{10,53}$ and where, as described earlier, kelch13 mutants, in the absence of partner drug resistance, are able to be cleared with adequate dose and duration of drug. ${ }^{7,47}$

\section{Which drug combinations are ideal for MDA in regions with multidrug resistance?}

The most desirable profile for antimalarial combination therapies has been described previously. ${ }^{36,54-56}$ Besides an exemplary safety profile, which is even more essential for drugs being used to treat individuals without clinical symptoms of malaria, some characteristics of an ideal combination therapy include a stable product given at high enough doses to remain effective even if resistance is present to the other drug, independent modes of action of the combined drugs with different metabolic targets, matching post-treatment drug activity profiles (based on elimination half-lives, dosage, and drug sensitivity), and if possible, drugs that are robust to the evolution of resistance (ie, high-level resistance evolves by a complex rather than simple mechanism) with antagonisticresistant mechanisms. An example of antagonistic-resistant mechanisms might be those for mefloquine and piperaquine. Mefloquine resistance is associated with increased copy number of the $p f m d r 1$ gene, ${ }^{57}$ while piperaquine resistance is associated with increased copy number of the plasmepsin II/ III genes, ${ }^{58,59}$ which tends to occur in the presence of a single copy $p f m d r 1 .{ }^{59}$ Consistent with this observation, geographic areas with highly prevalent piperaquine resistance have shown less prevalent mefloquine resistance, ${ }^{60,61}$ although a recent study suggests possible emergence of triple mutant parasites containing both amplified plasmepsin II and pfmdrl, as well as kelch 13 C580Y. ${ }^{62}$ The hypothesis of antagonistic resistance to these important partner drugs forms the rationale for triple combination therapy including an artemisinin derivative paired with both mefloquine and piperaquine. ${ }^{63}$ This triple therapy is now being tested in the GMS for treating clinical malaria, in hopes of preventing further emergence and spread of resistance. Such a combination may also be useful in deterring the emergence and spread of resistance in the context of MDA; however, further study of the long-term cardiac safety of this drug combination would be required before it could be used for this purpose. ${ }^{64}$ An alternative triple therapy could also be artemether-lumefantrine plus amodiaquine.

While long-acting ACTs are currently recommended for MDA, it is preferable that the ACT used for MDA should 
not be the same as the ACT being used as the first-line treatment for clinical malaria. ${ }^{11}$ Adding a drug to reduce malaria transmission, such as primaquine (to reduce transmissibility of $P$. falciparum gametocytes) or ivermectin (to reduce Anopheles mosquitoes), ${ }^{65-69}$ may also be desirable. Research is currently underway as part of the Ivermectin for Malaria in Southeast Asia (IMSEA) study to evaluate the safety of use of ivermectin in combination with primaquine and dihydroartemisinin-piperaquine. ${ }^{70}$

\section{Are certain drug-based elimination strategies likely to result in emergence and spread of resistance?}

MDA involves mass treatment of all, or a portion of, population regardless of the presence of symptoms of disease. As an alternative to MDA, which is administered without regard to infection status of individuals, screen-and-treat strategies only offer treatment to individuals who test positive for infection. Screen-and-treat strategies can be applied on a broader geographic scale (mass screening and treatment) or within smaller, more defined areas (focal screening and treatment). ${ }^{11}$ While MDA may result in a larger number of individuals being exposed to drug, because they are treated within a similar time frame, it provides some homogeneity in drug concentration profiles for the population. In a recent review, White ${ }^{52}$ suggested that such homogeneity may limit the spread of resistance, because drug concentrations are diminishing in the treatment community as a whole, such that a new infection from one individual in the treatment community that has gone through the vector stages is unlikely to encounter higher drug concentrations in another member of the treatment community. This would be particularly true in low transmission settings and during the low transmission season. Screen-and-treat approaches result in the treatment of only those individuals who test positive for infection, and individuals with infections with parasite density below the threshold of detection of the diagnostic being used for screening will not be treated, but may still contribute to transmission of new infections in the region (although if the diagnostic is highly sensitive, it is not clear whether the extremely low parasitemia infections left undetected will be transmissible). This scenario could lead to some heterogeneity in drug concentrations in the community and staggered windows of selection that might allow for selection of resistance. Future mathematical modeling studies may be helpful in exploring the impact of different types of drug-based elimination strategies on selection for drug resistance, particularly in areas with highly prevalent multidrug resistance.

\section{MDA and emergence of resistance in co-endemic pathogens}

Some drugs being considered for MDA in elimination programs that target malaria parasites or other organisms have effects on multiple pathogens. Although administration of such medications could have a synergistic effect in reducing the frequency of multiple diseases of public health importance, it may also exert drug pressure on organisms that are not the intended target of the elimination campaign. While this scenario would only be a concern in areas where these pathogens are co-endemic, this possibility should be taken into consideration when monitoring population prior to, during, and following MDA programs.

For example, the versatile endectocide ivermectin is being promoted as a promising tool for reduction of malaria transmission, ${ }^{67,71,72}$ as a result of its activity against the mosquito vector ${ }^{65,66,68,69}$ and potentially the malaria parasite itself. ${ }^{73}$ However, this drug is also of great importance for the control of lymphatic filariasis, onchocerciasis, and certain soil-transmitted helminths. Possible resistance to ivermectin has been reported for Onchocerca ${ }^{74}$ and for some helminths of veterinary importance. ${ }^{75}$ Although ivermectin has a different mechanism of action than insecticides commonly used against malaria vectors, Anopheles has the potential to develop resistance against ivermectin also. Surveillance of populations where ivermectin is used for malaria control and elimination activities should be ongoing to identify resistance, in either pathogens or the vector, should it arise. ${ }^{72}$

Another example of the potential of MDA to affect multiple pathogens is the use of artemisinin derivatives, as a complement to praziquantel, to treat schistosomiasis. A meta-analysis has indicated that artemisinin derivatives used in combination with praziquantel have the potential to increase cure rates when used to treat schistosomiasis. ${ }^{76}$ This effect is due to the activity of artemisinins against juvenile schistosomes, complementing the activity of praziquantel, which acts primarily on adult parasites. ${ }^{77}$ However, addition of artemisinin derivatives to MDA programs targeting Schistosoma in malaria-endemic areas has been controversial, ${ }^{76,78}$ owing to the potential to select for artemisinin resistance during such campaigns. Data are limited, and research is needed to understand the dynamics of an MDA regimen and co-infecting multiple organisms in developing effective strategies of malaria MDA. 


\section{MDA and Plasmodium vivax}

In geographical regions where $P$. vivax is co-endemic with P. falciparum, malaria elimination policy makers have also begun to consider pharmacotherapy active against $P$. vivax, particularly with regard to the hypnozoite reservoir. Currently, the WHO does not recommend MDA with primaquine to target the $P$. vivax hypnozoite reservoir or to interrupt $P$. vivax transmission when individual glucose-6-phosphate-dehydrogenase deficiency (G6PD) status remains unknown. ${ }^{11}$ Ongoing strategic discussion aimed at the interruption of $P$. vivax transmission includes consideration of chloroquine MDA, as well as the potential for primaquine MDA to be utilized after G6PD testing can be implemented. ${ }^{79,80}$ There is related consideration of whether and how MDA directed against $P$. falciparum in support of elimination may incidentally affect P. vivax transmission; ${ }^{81}$ however, more data are needed to reliably assess the impact of MDA targeting $P$. falciparum on the $P$. vivax reservoir and on its transmission dynamics.

\section{Conclusion}

In malaria elimination, the MDA approach is controversial, with well-recognized operational challenges and a suboptimal history of success. However, interest in MDA is on the rise, primarily driven by a fear of falciparum resistance to artemisinins and partner drugs, particularly in areas with a high risk of multidrug-resistant malaria. A sense of urgency to eliminate multidrug-resistant malaria has attracted the use of MDA as an elimination accelerator and to aid longterm malaria surveillance toward elimination, rather than a tool to interrupt malaria transmission single-handedly. As the urgency to eliminate malaria in the context of drug resistance prompts repeated attempts to design newer, better trials of MDA, these efforts ultimately culminate in the critical question of whether MDA may actually risk making the problem of drug resistance worse. Timely consideration of how MDA has affected drug resistance in the more recent past and may yet affect drug resistance in the future could be useful for catalyzing the implementation of more acceptable and effective MDA strategies in settings with rampant multidrug resistance.

MDA may be a useful strategy to eliminate malaria in isolated, low-transmission settings, particularly when combined with vector control or other measures to reduce malaria transmission, sufficient social mobilization, and sustained political support. This approach is being piloted in the GMS to eliminate multidrug-resistant falciparum malaria. While past MDA studies have provided some examples of the emergence of resistance with MDA, these were usually in cases where drug doses were subtherapeutic and/or parasites developed resistance against the drug being used by a simple mechanism. There has been little evidence to date that MDA has exacerbated the problem of drug resistance in areas plagued by multidrug resistance, but continued careful monitoring of clinical responses and molecular markers of resistance will be paramount. Use of therapeutic doses of carefully selected ACTs in asymptomatic individuals in the context of MDA implemented in low-transmission settings during the low transmission season may reduce the risk of emergence of resistance. Future studies, including mathematical modeling approaches, would be helpful in exploring the risk of promoting spread of drug resistance through MDA campaigns in areas with established resistance to artemisinins and their partner drugs.

\section{Disclosure}

The authors report no conflicts of interest in this work.

\section{References}

1. WHO. World Malaria Report, 2015. Geneva: WHO; 2015.

2. Gates BC, Chambers, R. From aspiration to action: what will it take to end malaria? Available from: http://endmalaria2040.org/assets/ Aspiration-to-Action.pdf. Accessed October 16, 2017.

3. Noedl H, Se Y, Schaecher K, et al. Evidence of artemisinin-resistant malaria in western Cambodia. $N$ Engl J Med. 2008;359(24): 2619-2620.

4. Dondorp AM, Nosten F, Yi P, et al. Artemisinin resistance in Plasmodium falciparum malaria. N Engl J Med. 2009;361(5):455-467.

5. Kyaw MP, Nyunt MH, Chit K, et al. Reduced susceptibility of Plasmodium falciparum to artesunate in southern Myanmar. PLoS One. 2013;8(3):e57689.

6. Hien TT, Thuy-Nhien NT, Phu NH, et al. In vivo susceptibility of Plasmodium falciparum to artesunate in Binh Phuoc Province, Vietnam. Malar J. 2012;11:355.

7. Ashley EA, Dhorda M, Fairhurst RM, et al. Spread of artemisinin resistance in Plasmodium falciparum malaria. $N$ Engl J Med. 2014;371(5):411-423.

8. WHO. Strategy for Malaria Elimination in the Greater Mekong Subregion (2015-2030). Geneva: WHO; 2015.

9. Takala-Harrison S, Jacob CG, Arze C, et al. Independent emergence of artemisinin resistance mutations among Plasmodium falciparum in Southeast Asia. J Infect Dis. 2015;211(5):670-679.

10. Miotto O, Amato R, Ashley EA, et al. Genetic architecture of artemisinin-resistant Plasmodium falciparum. Nat Genet. 2015;47(3): 226-234.

11. World Health Organization Evidence Review Group Meeting Report. Mass Drug Administration, Mass Screening and Treatment and Focal Screening and Treatment for Malaria. Geneva: World Health Organization; 2015.

12. Barber MA, Rice JB, Brown JY. Malaria studies on the Firestone rubber plantation in Liberia, West Africa. Am J Hyg. 1932;15(3):601-633.

13. Snowden FM. The Conquest of Malaria; Italy, 1900-1962. New Haven: Yale University Press; 2006.

14. Dixon DS. Paludrine (proguanil) as a malarial prophylactic amongst African labour in Kenya. East Afr Med J. 1950;27(3):127-130.

15. Molineaux L, Gramiccia G. The Garki Project: Research on the Epidemiology and Control of Malaria in the Sudan Savanna of West Africa. Geneva: World Health Organization; 1980. 
16. von Seidlein L, Walraven G, Milligan PJ, et al. The effect of mass administration of sulfadoxine-pyrimethamine combined with artesunate on malaria incidence: a double-blind, community-randomized, placebo-controlled trial in The Gambia. Trans $R$ Soc Trop Med Hyg 2003;97(2):217-225.

17. Kaneko A, Taleo G, Kalkoa M, Yamar S, Kobayakawa T, Björkman A. Malaria eradication on islands. Lancet. 2000;356(9241):1560-1564.

18. malERA Consultative Group on Drugs. A research agenda for malaria eradication: drugs. PLoS Med. 2011;8(1):e1000402.

19. Dola SK. Mass drug administration as a supplementary attack measure in malaria eradication programme. East Afr Med J. 1974;51(7):529-531.

20. von Seidlein L, Greenwood BM. Mass administrations of antimalarial drugs. Trends Parasitol. 2003;19(10):452-460.

21. Poirot E, Skarbinski J, Sinclair D, Kachur SP, Slutsker L, Hwang J. Mass drug administration for malaria. Cochrane Database Syst Rev. 2013;(12):CD008846.

22. Newby G, Hwang J, Koita K, et al. Review of mass drug administration for malaria and its operational challenges. Am J Trop Med Hyg. 2015;93(1):125-134.

23. Pinotti $M$. The addition of antimalarial drugs to cooking salt: its importance in malaria eradication programmes. Triangle. 1959;4:119-113.

24. D'Alessandro U, Buttiens H. History and importance of antimalarial drug resistance. Trop Med Int Health. 2001;6(11):845-848.

25. Eyles DE, Hoo CC, Warren M, et al. Plasmodium falciparum resistant to chloroquine in Cambodia. Am J Trop Med Hyg. 1963;12:840-8433.

26. Giglioli G, Rutten FJ, Ramjattan S. Interruption of malaria transmission by chloroquinized salt in Guyana, with observations on a chloroquineresistant strain of Plasmodium falciparum. Bull World Health Organ 1967;36(2):283-301.

27. Hall SA, Wilks NE. A trial of chloroquine-medicated salt for malaria suppression in Uganda. Am J Trop Med Hyg. 1967;16(4):429-442.

28. Plowe CV. The evolution of drug-resistant malaria. Trans $R$ Soc Trop Med Hyg. 2009;103(suppl 1):S11-S14.

29. Payne D. Did medicated salt hasten the spread of chloroquine resistance in Plasmodium falciparum? Parasitol Today. 1988;4(4):112-115.

30. Clyde DF, Shute GT. Resistance of Plasmodium falciparum in Tanganyika to pyrimethamine administered at weekly intervals. Trans $R$ Soc Trop Med Hyg. 1957;51(6):505-513.

31. Charles LJ, Van Der Kaay HJ, Vincke IH, Brady J. The appearance of pyrimethamine resistance in Plasmodium falciparum following selfmedication by a rural community in Ghana. Bull World Health Organ. 1962;26:103-108.

32. Denis MB, Tsuyuoka R, Lim P, et al. Efficacy of artemether-lumefantrine for the treatment of uncomplicated falciparum malaria in northwest Cambodia. Trop Med Int Health. 2006;11(12):1800-1807.

33. Denis MB, Tsuyuoka R, Poravuth Y, et al. Surveillance of the efficacy of artesunate and mefloquine combination for the treatment of uncomplicated falciparum malaria in Cambodia. Trop Med Int Health 2006;11(9):1360-1366.

34. Lwin KM, Imwong M, Suangkanarat P, et al. Elimination of Plasmodium falciparum in an area of multi-drug resistance. Malar J. 2015;14:319.

35. Hastings IM, Watkins WM, White NJ. The evolution of drug-resistant malaria: the role of drug elimination half-life. Philos Trans $R$ Soc Lond B Biol Sci. 2002;357(1420):505-519.

36. Nyunt MM, Plowe CV. Pharmacologic advances in the global control and treatment of malaria: combination therapy and resistance. Clin Pharmacol Ther. 2007;82(5):601-605.

37. Kay K, Hastings IM. Measuring windows of selection for anti-malarial drug treatments. Malar J. 2015;14:292.

38. Stepniewska K, White NJ. Pharmacokinetic determinants of the window of selection for antimalarial drug resistance. Antimicrob Agents Chemother. 2008;52(5):1589-1596.

39. Korsinczky M, Chen N, Kotecka B, Saul A, Rieckmann K, Cheng Q. Mutations in Plasmodium falciparum cytochrome $\mathrm{b}$ that are associated with atovaquone resistance are located at a putative drug-binding site. Antimicrob Agents Chemother. 2000;44(8):2100-2108.
40. Maude RJ, Nguon C, Dondorp AM, White LJ, White NJ. The diminishing returns of atovaquone-proguanil for elimination of Plasmodium falciparum malaria: modelling mass drug administration and treatment. Malar J. 2014;13:380.

41. Imwong M, Suwannasin K, Kunasol C, et al. The spread of artemisininresistant Plasmodium falciparum in the Greater Mekong subregion: a molecular epidemiology observational study. Lancet Infect Dis. 2017;17(5):491-497.

42. Leang R, Taylor WR, Bouth DM, et al. Evidence of Plasmodium falciparum malaria multidrug resistance to artemisinin and piperaquine in Western Cambodia: dihydroartemisinin-piperaquine open-label multicenter clinical assessment. Antimicrob Agents Chemother. 2015;59(8):4719-4726.

43. Spring MD, Lin JT, Manning JE, et al. Dihydroartemisinin-piperaquine failure associated with a triple mutant including kelch13 C580Y in Cambodia: an observational cohort study. Lancet Infect Dis. 2015;15(6):683-691.

44. Wernsdorfer WH. The biological and epidemiological basis of drug resistance in malaria parasites. Southeast Asian J Trop Med Public Health. 1992;23(suppl 4):123-129.

45. White NJ, Pongtavornpinyo W, Maude RJ, et al. Hyperparasitaemia and low dosing are an important source of anti-malarial drug resistance. Malar J. 2009;8:253.

46. Verdrager J. Epidemiology of emergence and spread of drug-resistant falciparum malaria in Southeast Asia. Southeast Asian J Trop Med Public Health. 1986;17(1):111-118.

47. Bethell D, SeY, Lon C, et al. Artesunate dose escalation for the treatment of uncomplicated malaria in a region of reported artemisinin resistance: a randomized clinical trial. PLoS One. 2011;6(5):e19283.

48. Hastings IM, Paget-McNicol S, Saul A. Can mutation and selection explain virulence in human P. falciparum infections? Malar J. 2004;3:2.

49. Phyo AP, Ashley EA, Anderson TJC, et al. Declining efficacy of Artemisinin combination therapy against $P$. falciparum malaria on the Thai-Myanmar Border (2003-2013): the role of parasite genetic factors. Clin Infect Dis. 2016;63(6):784-791.

50. World Wide Antimalarial Resistance Network (WWARN) Lumefantrine PK/PD Study Group. Artemether-lumefantrine treatment of uncomplicated Plasmodium falciparum malaria: a systematic review and meta-analysis of day 7 lumefantrine concentrations and therapeutic response using individual patient data. BMC Med. 2015;13:227.

51. Djimde AA, Doumbo OK, Traore O, et al. Clearance of drug-resistant parasites as a model for protective immunity in Plasmodium falciparum malaria. Am J Trop Med Hyg. 2003;69(5):558-563.

52. White NJ. Does antimalarial mass drug administration increase or decrease the risk of resistance? Lancet Infect Dis. 2017;17(1): e15-e20.

53. Straimer J, Gnadig NF, Witkowski B, et al. Drug resistance. K13-propeller mutations confer artemisinin resistance in Plasmodium falciparum clinical isolates. Science. 2015;347(6220):428-431.

54. Hastings IM, Hodel EM. Pharmacological considerations in the design of anti-malarial drug combination therapies - is matching half-lives enough? Malar J. 2014;13:62.

55. Burrows JN, van Huijsduijnen RH, Möhrle JJ, Oeuvray C, Wells TN. Designing the next generation of medicines for malaria control and eradication. Malar J. 2013;12:187.

56. Kremsner PG, Krishna S. Antimalarial combinations. Lancet. 2004;364(9430):285-294.

57. Peel SA, Bright P, Yount B, Handy J, Baric RS. A strong association between mefloquine and halofantrine resistance and amplification, overexpression, and mutation in the P-glycoprotein gene homolog (pfmdr) of Plasmodium falciparum in vitro. Am J Trop Med Hyg. 1994;51(5):648-658.

58. Amato R, Lim P, Miotto O, et al. Genetic markers associated with dihydroartemisinin-piperaquine failure in Plasmodium falciparum malaria in Cambodia: a genotype-phenotype association study. Lancet Infect Dis. 2017;17(2):164-173. 
59. Witkowski B, Duru V, Khim N, et al. A surrogate marker of piperaquineresistant Plasmodium falciparum malaria: a phenotype-genotype association study. Lancet Infect Dis. 2017;17(2):174-183.

60. Phuc BQ, Rasmussen C, Duong TT, et al. Treatment failure of dihydroartemisinin/piperaquine for Plasmodium falciparum Malaria, Vietnam. Emerg Infect Dis. 2017;23(4):715-717.

61. Rasmussen C, Ariey F, Fairhurst RM, et al. Role of K13 mutations in artemisinin-based combination therapy. Clin Infect Dis. 2016;63(12): 1680-1681.

62. Rossi G, De Smet M, Khim N, et al. Emergence of Plasmodium falciparum triple mutant in Cambodia. Lancet Infect Dis. 2017;17(12):1233.

63. Maxmen A. Back on TRAC: new trial launched in bid to outpace multidrug-resistant malaria. Nat Med. 2016;22(3):220-221.

64. Chotsiri P, Wattanakul T, Hoglund RM, et al. Population pharmacokinetics and electrocardiographic effects of dihydroartemisinin-piperaquine in healthy volunteers. Br J Clin Pharmacol. 2017;83(12):2752-2766.

65. Tesh RB, Guzman H. Mortality and infertility in adult mosquitoes after the ingestion of blood containing ivermectin. Am J Trop Med Hyg. 1990;43(3):229-233.

66. Chaccour C, Lines J, Whitty CJ. Effect of ivermectin on Anopheles gambiae mosquitoes fed on humans: the potential of oral insecticides in malaria control. J Infect Dis. 2010;202(1):113-116.

67. Foy BD, Kobylinski KC, da Silva IM, Rasgon JL, Sylla M. Endectocides for malaria control. Trends Parasitol. 2011;27(10):423-428.

68. Kobylinski KC, Deus KM, Butters MP, et al. The effect of oral anthelmintics on the survivorship and re-feeding frequency of anthropophilic mosquito disease vectors. Acta Trop. 2010;116(2):119-126.

69. Kobylinski KC, Sylla M, Chapman PL, Sarr MD, Foy BD. Ivermectin mass drug administration to humans disrupts malaria parasite transmission in Senegalese villages. Am J Trop Med Hyg. 2011;85(1):3-5.

70. Kobylinski K. Safety and mosquito-lethal efficacy of ivermectin, dihydroartemisinin-piperaquine, and primaquine: Ivermectin for Malaria in Southeast Asia (IMSEA Study, Thailand). In: American Society of Tropical Medicine and Hygiene Annual Meeting; Atlanta, GA; 2016.
71. Omura S, Crump A. Ivermectin and malaria control. Malar J. 2017;16(1):172.

72. Chaccour CJ, Kobylinski KC, Bassat Q, et al. Ivermectin to reduce malaria transmission: a research agenda for a promising new tool for elimination. Malar J. 2013;12:153.

73. Panchal M, Rawat K, Kumar G, et al. Plasmodium falciparum signal recognition particle components and anti-parasitic effect of ivermectin in blocking nucleo-cytoplasmic shuttling of SRP. Cell Death Dis. 2014;5:e994.

74. Osei-Atweneboana MY, Awadzi K, Attah SK, Boakye DA, Gyapong JO, Prichard RK. Phenotypic evidence of emerging ivermectin resistance in Onchocerca volvulus. PLoS Negl Trop Dis. 2011;5(3):e998.

75. Smits HL. Prospects for the control of neglected tropical diseases by mass drug administration. Expert Rev Anti Infect Ther. 2009;7(1):37-56.

76. Pérez del Villar L, Burguillo FJ, López-Abán J, Muro A. Systematic review and meta-analysis of artemisinin based therapies for the treatment and prevention of schistosomiasis. PLoS One. 2012;7(9):e45867.

77. Utzinger J, Xiao SH, Tanner M, Keiser J. Artemisinins for schistosomiasis and beyond. Curr Opin Investig Drugs. 2007;8(2):105-116.

78. Utzinger J, Tanner M, Keiser J. ACTs for schistosomiasis: do they act? Lancet Infect Dis. 2010;10(9):579-581.

79. Hsiang M, Hwang J, Tao A, et al. Mass drug administration for the control and elimination of Plasmodium vivax malaria: an ecological study from Jiangsu province, China. Malar J. 2013;12:383.

80. Robinson LJ, Wampfler R, Betuela I, et al. Strategies for understanding and reducing the Plasmodium vivax and Plasmodium ovale hypnozoite reservoir in Papua New Guinean Children: a randomised placebocontrolled trial and mathematical model. PLoS Med. 2015;12(10): e1001891.

81. Landier J, Kajeechiwa L, Thwin MM, et al. Safety and effectiveness of mass drug administration to accelerate elimination of artemisininresistant falciparum malaria: a pilot trial in four villages of Eastern Myanmar. Wellcome Open Res. 2017;2:81.
Infection and Drug Resistance

\section{Publish your work in this journal}

Infection and Drug Resistance is an international, peer-reviewed openaccess journal that focuses on the optimal treatment of infection (bacterial, fungal and viral) and the development and institution of preventive strategies to minimize the development and spread of resistance. The journal is specifically concerned with the epidemiology of antibiotic

\section{Dovepress}

resistance and the mechanisms of resistance development and diffusion in both hospitals and the community. The manuscript management system is completely online and includes a very quick and fair peerreview system, which is all easy to use. Visit http://www.dovepress.com/ testimonials.php to read real quotes from published authors. 